\title{
LA AUTORIDAD LABORAL EUROPEA: PRESENTACIÓN DE LA PROPUESTA DE LA COMISIÓN EUROPEA DE 13 DE MARZO DE 2018
}

\author{
Miguel Cuenca Alarcón \\ Departamento de Derecho del Trabajo y de la Seguridad Social \\ Facultad de Derecho. Universidad Complutense de Madrid \\ miguelcu@pdi.ucm.es
}

\section{INTRODUCCIÓN}

El reconocimiento de derechos sociales está vinculado con la creación de organismos públicos que conforman lo que comúnmente conocemos como Administración laboral; organismos que garantizan tales derechos a través de actividades de prestación de servicios, como la Seguridad Social, la intermediación laboral o la formación; de control, policía o inspección; de fomento e incentivo, o de conciliación, mediación y arbitraje, por citar las más conocidas ${ }^{1}$. Esta afirmación es válida en el ámbito estatal y lo es también en la Unión Europea, aunque con distinto y menor alcance. Desde ciertos puntos de vista podría decirse también que la Administración europea es menos visible que las nacionales. Se trata, sin embargo, de una pareja o binomio inseparable, especialmente cuando en el plano social y económico los agentes o actores en presencia actúan a nivel europeo, de ahí el interés de la reciente propuesta para la creación de una Autoridad Laboral Europea (en adelante, ELA por sus siglas en inglés); propuesta presentada en marzo del presente año por la Comisión Europea. Como expondré a continuación, se trata de un organismo que asumiría buena parte de las funciones que actualmente desempeñan otros órganos de la Unión y que, al mismo tiempo, potenciaría la actuación coordinada de las

${ }^{1}$ El papel de la Administración laboral tiende en nuestro sistema a clasificarse en siete grandes ámbitos: formación profesional, seguridad social, seguridad y salud, servicios de empleo, relaciones colectivas, igualdad e inspección de trabajo, desarrollando esta última también funciones mediadoras y de arbitraje. 
distintas Administraciones nacionales en supuestos de movilidad transnacional. Sin dejar de hacer crónica legislativa, intercalaré también en los apartados sucesivos algunas ideas básicas sobre el significado de la creación de una autoridad laboral.

Es notorio el vínculo que existe entre esta y otras propuestas de reforma con el desarrollo del denominado «pilar social» proclamado en noviembre del pasado año en la cumbre social de Goteburgo en favor del empleo justo y el crecimiento ${ }^{2}$. Pocos meses antes vio la luz formalmente la propuesta de creación de la ELA en el discurso de la Unión del presidente de la Comisión, suscitando importantes expectativas entre los distintos agentes sociales y la doctrina. Desde distintos ámbitos se valoró la posibilidad de dotarla de funciones directamente operativas, preceptivas y vinculantes en terrenos que iban desde la ya consolidada difusión de información sobre legislación y prácticas de empleo asociadas a la movilidad, pasando por el apoyo al desarrollo de comités de empresa europeos o instrumentos de representación en la sociedad europea, incluyendo también la creación de una Inspección de Trabajo u órgano análogo a nivel europeo, y considerando también la creación de un sistema de mediación, conciliación y arbitraje que, en su caso, permitiera o apoyara el desarrollo de un sistema de negociación colectiva europeo ${ }^{3}$. Creo que estas expectativas señalan cuál es el camino que debe recorrerse para afianzar el pilar social, al menos en los ámbitos del trabajo europeo transnacional, y tal recorrido puede considerarse ya un deber u obligación del vigente Derecho europeo. Aunque, como veremos, la propuesta final presentada por la Comisión no cumple todas ellas, optando básicamente por reforzar la colaboración interestatal y la integración de funciones preexis-

${ }^{2}$ Sobre los distintos hitos que culminan con la proclamación del pilar social me remito a E. Rojo TORRECILLA, «El pilar europeo de derechos sociales. Especial referencia a la importancia de los derechos de negociación y acción colectiva», disponible en http://www.eduar dorojotorrecilla.es/2017/11/el-pilar-europeo-de-derechos-sociales.html.

3 Me remito, por todos, a los informes y documentos que acompañan a la propuesta de la Comisión, así como a M. VelázQueZ, «La vigilancia y control de la Inspección de Trabajo y Seguridad Social sobre los desplazamientos transnacionales en el ámbito de la UE-EEE», Revista del Ministerio de Empleo y Seguridad Social, núm. 128 (2017), pp. 247 y ss.; S. FERNANDES, «What is our Ambition for the European Labor Authority?», Policy Paper 219, Institute Jacques Delors, Berlin, 2018, disponible en http://www.delorsinstitut.de/2015/wpcontent/uploads/2018/03/20180309_European-Labour-Agency_Fernandes.pdf; J. CREMERS, «Towards an European Labour Authority. Mandate, Main Tasks and Open Questions», Friedrich-Ebert-Stiftung, 2018, y Z. RASNACA y R. JAGODZINSKI, «Giving Juncker's Proposed European Labour Authority Real Clout», Social Europe, 2017, disponible en bttps://Www. Etui.Org/Fr/Actualites/Giving-Juncker-S-Proposed-European-Labour-Authority-Real-Clout. 
tentes, se trata de una propuesta abierta cuyo desarrollo puede conllevar una mejora de los procedimientos de control disponibles y una facilitación del diálogo social europeo, que en un futuro no muy lejano justifique nuevas reformas para avanzar en la línea de horizonte comentado. Como veremos a continuación no son pocas las reformas paralelas pendientes en materia de movilidad transnacional, reformas que pueden ser un vehículo para potenciar el pilar social ${ }^{4}$.

${ }^{4}$ Junto a las anteriores referencias me remito, por todos, a M. ARETA MartíneZ, «Coordinación de los sistemas europeos de Seguridad Social y exportación de la prestación por desempleo tras la aplicación del Reglamento (CE) núm. 883/2004», en El futuro europeo de la protección social, Murcia, Laborum, 2010, pp. 167-189, disponible en www.aesss.org/ wp-content/uploads/2010/02/Com.III_.Areta_doc; J. GarCÍA MuRCIA (dir.), Condiciones de empleo y relaciones de trabajo en el Derecho de la Unión Europea. Un estudio de jurisprudencia del Tribunal de Justicia, Cizur Menor, Aranzadi, 2018; íD. (dir.), Libertad de circulación $y$ derechos de protección social en la Unión Europea. Un estudio de jurisprudencia del Tribunal de Justicia, Madrid-Oporto, Juruá, 2016; M. E. CASAS BAAMONDE, Los desplazamientos temporales de trabajadores en la Unión Europea y en el Espacio Económico Europeo, Madrid, Civitas, 2001; A. DesDentado Bonete, «Trabajadores desplazados y trabajadores fronterizos en la Seguridad Social europea: del Reglamento 1408/1971 al Reglamento 883/2004», Revista del MTAS, núm. 64 (2006), pp. 19 y ss.; O. Fotinopoulou BASURKo (coord.), El desplazamiento de trabajadores en el marco de la Unión Europea: presente y futuro, Barcelona, Atelier, 2017; A. GuAMÁn HeRnÁNDEZ, «La propuesta de reforma de la Directiva 96/71 de desplazamiento de trabajadores en el marco del plan de trabajo de la Comisión Europea para el 2016», Revista de Derecho Social, núm. 73 (2016), pp. 113-126; E. LóPez TeRRADA, «La Ley aplicable en materia de Seguridad Social a las relaciones laborales internacionales: las normas comunitarias de coordinación», en AAVV, La internacionalización de las relaciones laborales, Valencia, Tirant lo Blanch, 2017, pp. 183-226; M. LlobERA VILA, «El art. 1.4 ET a la luz de la jurisprudencia comunitaria en materia de ley aplicable al contrato internacional», Revista de Derecho Social, núm. 73 (2016), pp. 127-148; J. L. Monereo PéreZ, «Los derechos de Seguridad Social de los trabajadores migrantes: inmigración laboral y refugiados», Revista de Derecho Migratorio y Extranjería, núm. 41 (2016), pp. 409 y ss.; P. PÁramo MonTERO, «La nueva Directiva 2014/67/UE sobre desplazamiento temporal de trabajadores», Trabajo y Derecho, núm. 3 (2015), pp. 52-70; I. A. Rodríguez Cardo, en C. SÁnCHEZ RoDAs NAVARRo (dir.), La coordinación de los sistemas de Seguridad Social. Los Reglamentos 883/2004 y 987/2009, Murcia, Laborum, 2010; M. RodRíGUEZ-PiÑERO y BRAVO-FERRER., «El desplazamiento temporal de trabajadores y la Directiva 2014/67 relativa a la garantía de cumplimiento de la Directiva 96/71/CEE», Derecho de las Relaciones Laborales, núm. 5 (2016), pp. 407-416; Y. SÁnchez URAn-Azaña, «Ciudadanía de la Unión Europea y derechos de protección social comunitaria. Balance y perspectivas del modelo condicional de solidaridad social comunitaria», Revista Española de Derecho Europeo, núm. 56 (2015), pp. 43-88; R. VAN DER STRATEN, The Revision of the Posted Workers Directive: High Hopes or Empty Promises in the Search for a Stronger Social Dimension?, Leiden, Leiden University, 2017, disponible en www.nver.nl/s/scriptie-R-van-der-Straten-2017-4ene.pdf, y S. SCHWARZ y M. KIELBASA, «Posted or Sent? In the Context of the Revision of Social Security Coordination Regulations», Labour Mobility Initiative, 2018, disponible en https://www.mobilelabour.eul. 


\section{EL INDISPENSABLE DESARROLLO DE UN DERECHO SOCIAL DE COORDINACIÓN ADMINISTRATIVA, INTEGRACIÓN Y GARANTÍA NORMATIVA}

Entre los elementos que hacen del desarrollo de la ELA una aspiración tan oportuna como inaplazable para afianzar no solo la visibilidad, sino también la acción de la Unión en materia social se encuentra, como hemos visto al inicio, la indisoluble conexión o incluso identificación entre derechos sociales y garantías administrativas y materiales. Como es sabido, en el ámbito laboral y de Seguridad Social el desarrollo de la normativa europea ha supuesto - sin lugar al error ya a estas alturas- el reconocimiento y consagración de derechos sociales fundamentales que han entrado y entran en continuo diálogo con los ordenamientos nacionales para servir de guía del cambio y que actúan como vía de recepción inicial de los Convenios de la Organización Internacional del Trabajo (en adelante, OIT). Este diálogo supone también desde un inicio la salvaguardia de derechos económicos vinculados a las conocidas como libertades fundamentales - libre circulación de personas, servicios, mercancías y capitales-, que, sin perjuicio de las situaciones de conflicto bien conocidas ${ }^{5}$, se acompaña ya, como digo, de un trasfondo social entre cuyos hitos se encuentra la consagración de derechos como la igualdad y no discriminación en las relaciones laborales, la participación en procesos de reestructuración, los derechos de información y consulta, la protección de la seguridad y salud en el trabajo o la protección en casos de desplazamiento, incluyendo la coordinación de los servicios propios de la Seguridad Social.

Las principales técnicas utilizadas para desarrollar el Derecho social de la Unión no se concretan en la uniformización de las regulaciones aplicables ni, como regla, en la creación de una administración común, sino en la conocida armonización en el ámbito laboral, por un lado, y, por otro, en la coordinación de las legislaciones en el ámbito de la Seguridad Social; coordinación que ha alcanzado también a las correspondientes Administraciones nacionales. A través de la primera técnica - la armonización - la norma europea ha establecido estándares mínimos vinculantes,

5 Sobre las distintas vertientes de esta complejidad puede verse el artículo de A. BAYLOS GRAU, «El espacio supranacional de ejercicio del derecho de huelga y la restricción legal de sus capacidades de acción», Revista de Derecho Social, núm. 41 (2008), pp. 123 y ss. Vid. también íD., «Derecho sindical, gestión sindical del conflicto y crisis económica», Revista de Derecho Sindical, núm. 66 (2014), pp. 13 y ss. 
susceptibles de ser desarrollados en diversos sentidos; a través de la segunda técnica, la Unión ha permitido que los Estados mantengan y desarrollen sus políticas propias de Seguridad Social, pero asumiendo que deben entrar en contacto e integrar sus sistemas en casos de desplazamientos o migraciones para garantizar la protección dispensada por cada Estado, permitiendo su acumulación o totalización para generar pensiones, subsidios, asistencia sanitaria u otras prestaciones. Aquí, en la Seguridad Social, no hay estándares mínimos —o no son amplios ni explícitos-, sino una regulación que permite aplicar regímenes de distintos Estados de forma conjunta si trabajadoras o empresas se desplazan a Estados distintos ${ }^{6}$. En el contexto de estas técnicas, la regulación europea opta, en la mayoría de los casos, por atribuir a la Administración laboral de cada Estado miembro el poder de hacer cumplir la normativa laboral armonizada y de Seguridad Social objeto de coordinación en su propio territorio, sin perjuicio del deber de cooperación de otros Estados y de la ayuda de la labor interpretativa del Tribunal de Justicia de la Unión Europea (en adelante, TJUE) sobre el alcance del Derecho europeo. En otros casos, sin embargo, dicha regulación ha optado por atribuir una cierta prevalencia inicial a la Administración de un concreto Estado miembro frente a las demás.

Existen ámbitos donde el elemento europeo hace necesario, por no decir imprescindible, la entrada en escena de una autoridad laboral común, a modo de órgano de coordinación de la Unión, sin perjuicio de los matices que puedan exponerse ${ }^{7}$. Pero no deja de ser menos necesaria si se parte de la necesidad de preservar los propios derechos sociales, pues pueden surgir diferencias de criterio entre las distintas Administraciones implicadas en relación con una misma y única relación laboral (trabajador o trabajadora) o una misma y única empresa. Tampoco resulta apropiado atribuir competencias a un único Estado en lugar de optar por el reparto, dadas las dificultades que existen para desarrollar determinadas funciones

${ }^{6}$ Asumo la propuesta lingüística de designar en femenino el sector de la población compuesto mayoritariamente de mujeres. En el ámbito laboral o, más en general, del empleo —incluyendo el empleo por antonomasia, el doméstico—, sin más adjetivos, las mujeres son mayoría.

${ }^{7} \mathrm{La}$ pertinencia de estos órganos $-\mathrm{O}$ procedimientos comunes- puede justificarse también por contraste con otros ámbitos vinculados con la acción administrativa, como aprecia Cremers, en el sentido de que no hay nada comparable en el ámbito laboral a los procedimientos de queja o reclamación desarrollados en el ámbito de la infracción de reglas sobre competencia, así como para tratar las quejas y audiencias con las partes concernidas. Vid. J. CREMERS, «Towards an European Labour Authority...», op. cit., p. 6. Ello exigiría una reforma de más alcance. 
administrativas y de control e inspección desde centros lejanos, es decir, a distancia, desde otros Estados, o atribuir poderes de policía a nacionales desplazados de terceros Estados.

Es cierto que las anteriores diferencias y dificultades pueden tratar de resolverse, como se viene haciendo, a través de la comunicación y cooperación entre autoridades o, en última instancia, a través de decisiones judiciales que pueden aplicar normas de conflicto - informadas, en su caso, por una sentencia del TJUE-. También lo es que actualmente existen diversos organismos que tratan de llevar a cabo una labor de mediación entre autoridades, que es precisamente - o sería - una de las funciones esenciales de la ELA. Pero la visibilidad y la eficacia de la acción de estos últimos organismos no es un dato de partida ${ }^{8}$; ni tampoco es del todo clara la regulación de su estructura, funciones y procedimientos. En la práctica, uno de los problemas detectados en la cooperación interadministrativa es la dilación de las soluciones, así como la ausencia de respuestas efectivas frente a los incumplimientos que se detectan ${ }^{9}$. En este sentido, se ha podido identificar entre las principales lagunas de la legislación europea actual la dualidad de trámites concurrentes desde un punto de vista laboral y de Seguridad Social ${ }^{10}$, la falta de complitud de la regulación de los procedimientos de control estatales — significativamente en la expedición del certificado $\mathrm{A} 1^{11}$ - y la falta de mecanismos de resolución de disputas preceptivos y vinculantes entre Estados sobre la legislación aplicable ${ }^{12}$.

${ }^{8}$ Fernandes habla también de «debilidad» de la cooperación entre autoridades que ha permitido, según constata, la explotación laboral y la competencia en condiciones ilegales. Vid. S. FERNANDES, «What is our Ambition for the European Labor...», op. cit., p. 3.

9 J. CREmers, «Towards an European Labour Authority...», op. cit., p. 6.

${ }_{10}$ Como los documentos de comunicación de desplazamiento, desde el punto de vista laboral, y de legislación de Seguridad Social aplicable o A1, desde el punto de vista de la Seguridad Social. Vid. cfr. M. VelázquEZ, «La vigilancia y control de la Inspección de Trabajo...», op. cit., pp. 270 y ss.

${ }^{11}$ La propuesta de reforma del Reglamento 883/2004, Estrasburgo, 13 de diciembre de 2016, COM (2016) 815 final, 2016/0397 (COD), atribuye a la Comisión poderes de ejecución para desarrollar la expedición, el formato y el contenido del documento portátil (A1) que acredita la legislación en materia de Seguridad Social aplicable al titular, la determinación de las situaciones en que se expedirá el documento, los elementos que deben verificarse antes de que pueda expedirse el documento y la retirada del documento cuando su exactitud y su validez sean impugnadas por la institución competente del Estado miembro de empleo.

${ }^{12}$ Cfr. M. Velázquez, «La vigilancia y control de la Inspección de Trabajo...», op. cit., pp. 270 y ss. Respecto de las necesidades vinculadas con la colaboración de las Inspecciones de Trabajo y entre estas y la Administración, me remito a las conclusiones del proyecto CIBELES desarrollado en el seno del CARIT. Disponible en http://www.empleo.gob. 
$\mathrm{Al}$ margen de estos déficits, interesa destacar que el tratamiento de la actividad transnacional hace que la labor de gestión, control e inspección sea ya, de hecho, objeto en sí misma de una cierta armonización a nivel europeo - como acontece con la expedición de certificados de Seguridad Social A1-, de manera que los Estados miembros vienen actualmente obligados no solo a ceder en favor de otros Estados sus facultades de control, sino que deben ejercer su actividad administrativa conforme a los procedimientos de coordinación que determina la Unión. Creo, además, que es lógico asumir que la idea de coordinación debe alcanzar también la definición de la actuación administrativa y de control o inspección en el ámbito de la Seguridad Social u otras vinculadas. Ello no es incompatible con la afirmación de las insuficiencias de la regulación vigente, claramente identificadas en la doctrina ${ }^{13}$, sino, bien al contrario, es una manera de resaltar que el modelo de actuación administrativa hasta ahora asumido, frente a otros posibles, presenta importantes problemas, pero no por ello deja de incidir en la actividad administrativa de los Estados miembros.

Con ello, de un modo u otro, se define el modelo europeo de gestión e inspección de las relaciones de trabajo para estos casos de movilidad. Y con ello se decide si las relaciones de trabajo transnacionales merecen o no una administración coherente, por decirlo de forma gráfica. Es decir, admitida la necesidad de facilitar la movilidad, la definición del modelo puede prestar más atención a ese objetivo básico o a los problemas vinculados a la distribución de competencia interestatal, o bien, en fin, y en lo que más interesa, a la tutela y protección de empresas, trabajadoras y trabajadores, cuando tales objetivos o necesidades entran en conflicto, en conexión, en su caso, con la constatación de la debilidad presupuestaria y de recursos de algunas Administraciones estatales. $\mathrm{O}$, dicho de otro modo, se hace necesario crear un nivel y procedimientos de gestión administrativos que eviten —en la medida de lo posible_ o compensen tendencialmente los peligros de las dilaciones o la ineficacia de una inspección y gestión repartida entre varios órganos estatales, así como de los correspondientes órganos judiciales y los propios de la Unión ${ }^{14}$.

es/itss/web/Sala_de_comunicaciones/Noticias/Archivo_Noticias/2011/11/20111122_not_ web_port.html.

${ }_{13}$ M. VelázQuez, «La vigilancia y control de la Inspección de Trabajo...», op. cit., p. 270 .

${ }^{14}$ En relación con la configuración de los procesos judiciales actuales de la Unión me remito a R. Alonso GarCía, «Lisboa y el Tribunal de Justicia de la Unión Europea», en Derecho de la Unión Europea y Tratado de Lisboa, Madrid, Civitas, 2013, pp. 307 y ss. 
Si ello es así, la creación de una autoridad europea no solo debe explicarse como un medio para lograr una movilidad acorde con el Derecho de la Unión, sino también para conseguir condiciones justas de empleo. El énfasis en esta última garantía puede ser, así, una vía más propicia para lograr un tratamiento inclusivo de la movilidad, evitando un juicio negativo sobre la misma, que no es, en cuanto tal, el elemento determinante del reproche jurídico, sino el incumplimiento de estándares laborales mínimos que tratan de alcanzar a todas las trabajadoras y trabajadores de la Unión tanto respecto de las condiciones de trabajo en el país de destino como en el ejercicio de otros derechos individuales y colectivos. En este contexto, la norma espera que se cumplan los estándares del país de destino y no niega ni limita la movilidad, de manera que cualquier reproche a la empresa no debería alcanzar a la prestación de trabajo considerada: corresponde aplicar el ordenamiento laboral y de Seguridad Social del país de destino. Si existe la posibilidad de concebir la respuesta jurídica en estos términos es preferible presentarla tal y como es para lograr desde la propia nomenclatura legal la cohesión europea, aunque en otros contextos —estancias en situaciones de ausencia de empleo y rentas, por ejemplo- quepa todavía debatir cómo promover esta cohesión.

Puede considerarse, adicionalmente, que frente a una dialéctica de oposición entre Estados, Estados-Comisión o entre particulares asociada a una posible dilación y a procesos judiciales, como la cuestión prejudicial o de incumplimiento, parece más adecuado optar por conducir la actividad de control y gestión por sus propios cauces, es decir, sin rebasar tendencialmente el ámbito administrativo, fomentando respuestas coordinadas inmediatas típicas de la Administración laboral y de las correspondientes Inspecciones de Trabajo. Es decir, una coordinación administrativa ex ante. Algunos de estos objetivos se están tratando de alcanzar con la reforma de los Reglamentos de referencia, singularmente del 883/2004, donde se prevén procedimientos sumarios de comunicación entre autoridades, si bien no es claro que se garantice la cercanía e inmediatez de la actuación administrativa, de ahí la importancia de buscar actuaciones integradas —en el ámbito de la ELA, por ejemplo—y, al mismo tiempo, descentralizadas ${ }^{15}$.

15 Junto con la bibliografía ya citada me remito a las consideraciones que expreso en M. CuenCa AlarCón, «Seguridad Social, libre circulación y mecanismos de coordinación en casos de desplazamiento temporal: jurisprudencia, reformas, debates. Sentencia del Tribunal de Justicia de la Unión Europea (Gran Sala) de 6 de febrero de 2018, asunto C-359/16, Altun», La Ley-Unión Europea, núm. 58 (2018). 
En realidad, esta ambición y este desafío pueden ser similares, por más que no idénticos, a los que se plantean en otros ámbitos laborales, como anticipa la propuesta de la Comisión que ahora nos ocupa, al incluir entre los posibles terrenos de actuación los supuestos de disrupción o, si se prefiere, transformación o reestructuración empresarial de tipo transfronterizo. Es posible recordar que Directivas como la 96/71/CE — recientemente modificada por la Directiva 2018/957, de 28 de junio de 2018-; la 2014/67/UE, en materia de desplazamiento de trabajadoras y trabajadores - considerada de forma explícita en la propuesta de la ELA por la Comisión-; la 2001/23/CE, en materia de transmisión de empresas, o, sin agotar las referencias, la Directiva 98/59/CE, en materia de despidos colectivos, han planteado y plantean cuestiones cuyo tratamiento a través de la armonización resulta muy complejo, al tiempo que atractivo por su valor de referente de coordinación: ¿cómo garantizar el derecho a la negociación colectiva cuando la plantilla trabaja habitualmente en dos Estados miembros? ¿Cómo garantizar la intervención mediadora de la Administración u otros órganos en caso de conflicto, derivado o no de la negociación? En algunas sentencias del TJUE bien conocidas (asuntos Viking, Laval o, entre otros, Comisión-Luxemburgo ${ }^{16}$ y en buena parte de la doctrina, la cuestión se aborda, habitualmente, desde la perspectiva de la ponderación de la libre circulación de personas y libre prestación de servicios frente al derecho colectivo de que se trate (huelga, conflicto, negociación colectiva, etc.), valorando si la forma en que son ejercidos estos derechos colectivos imponen o no cargas excesivas o están justificados por razones de interés general. Con ello se está perfilando - como acontece con la Administración laboral - un cierto modelo legal de negociación colectiva caracterizado aquí, nuevamente, por las lagunas concurrentes; por una cierta prevalencia de los foros de negociación del país de origen, que se acompaña con la apertura a una negociación voluntaria en el país de destino no sometida, a primera vista, al deber de negociar, y con una instrumentación y eficacia incierta, dado que no se definen las vías para integrarse en los mecanismos de negociación ordinaria del país de destino ni la eficacia de lo negociado en el país de destino respecto del país de origen ${ }^{17}$. Es algo que también

16 SSTJUE de 11 de diciembre de 2007, asunto C-438/05; de 18 de diciembre de 2007, asunto C-341/05, o de 19 de junio de 2008, asunto C-319/06.

17 Dada la competencia asumida por la Unión en materia de defensa y representación de los intereses de los trabajadores, parece difícil pensar el ejercicio de derechos colectivos en los términos propios de los derechos políticos. Los Tratados reconocen derechos políticos en el ámbito de la Unión y respecto de las elecciones municipales, y no abordan de 
acontece en la propia Directiva 96/71/CE, donde la garantía de aplicación de convenios con eficacia general del país de destino no se conecta con la posibilidad de participar en dicha negociación, presuponiéndose el ejercicio de derechos colectivos en el país de origen e incluso, quizá, la imposibilidad de negociar en el país de destino debido a la temporalidad del desplazamiento. Pero no se excluye esta negociación —ni expresamente por la norma ni por la jurisprudencia citada que asume su ejercicio voluntario- ni tampoco, en su caso, el ejercicio del derecho de huelga, que puede ser también el origen del pacto ${ }^{18}$. La modificación de 2018 de la Directiva 96/71/CE no ha abordado expresamente estas cuestiones, por más que haya introducido importantes cambios respecto de la propuesta inicial, singularmente en lo que respecta al periodo de aplicación de la legislación de origen, que se limita como regla a un año ${ }^{19}$.

Ciertamente, las competencias europeas en materias colectivas como las citadas son limitadas o inexistentes, según el caso, desde el punto de vista de la armonización. Pero también lo es, por un lado, que el desarrollo y aplicación del ordenamiento comunitario debe atenerse a los derechos consagrados en la Carta Europea de Derechos Fundamentales, y, por otro, que cualquier empeño en facilitar la libre circulación de trabajo y servicios exige considerar las necesidades de conciliar el ejercicio simultáneo y sucesivo no solo de derechos individuales, sino también colectivos, si es que pueden pensarse los unos sin los otros. Aunque puedan analizarse como normas de conflicto, en realidad son también normas que tratan o tratarían de asegurar la representación y defensa colectivas y cuya definición no se

forma expresa la concreción de esos derechos políticos en el ámbito estatal, de forma tal que no reconocen derechos políticos adicionales, sin perjuicio de la garantía del Estado democrático de Derecho. Parece que algo similar ocurre con la negociación colectiva —o la huelga-, pero aquí sí existe una llamada expresa a la armonización en materia de defensa y representación colectiva de intereses que, indirectamente, puede conectarse con la negociación colectiva. Por otro lado, no es posible negar genéricamente el ejercicio de derechos sindicales en ningún territorio de la Unión.

${ }^{18}$ Indica E. Rojo TorRecilla, «El pilar europeo de derechos sociales...», op. cit., p. 1, que el ejercicio del derecho de huelga parece finalmente quedar vinculado a la libertad de establecimiento, dado el cúmulo de limitaciones concurrentes en la jurisprudencia; ello no impide que nuestro Derecho y la propia normativa europea no establezcan limitaciones explícitas al respecto; limitaciones que, por otro lado, serían de muy difícil justificación, creo que imposible.

${ }_{19}$ Sin perjuicio de ulteriores matices, la versión final de la modificación de la Directiva $96 / 71$ ha tenido en cuenta algunas observaciones sindicales asumidas también por la doctrina. El carácter temporal variable de la aplicación de la legislación de destino lo he asumido también como propuesta respecto de la Seguridad Social en M. Cuenca Alarcón, «Seguridad Social, libre circulación y mecanismos de coordinación...», op. cit., pp. 9 y ss. 
debe diferir al desarrollo expreso de un sistema de negociación colectiva transnacional, pues la necesaria coordinación entre las prácticas negociales no presupone un convenio colectivo de ámbito europeo, sino un sistema de coordinación de convenios y procesos negociales de varios ámbitos nacionales, por más que uno y otro desarrollo puedan ir de la mano y adoptar soluciones simétricas ${ }^{20}$. $\mathrm{O}$, dicho de otro modo, más que armonizar se trata de coordinar, sin perjuicio de que esta coordinación pueda comportar un cierto desarrollo de un modelo negocial, como acontece con el desarrollo del modelo de control de la Seguridad Social. Es algo que ya se hace también en un plano individual cuando se afirma, por ejemplo, que en casos de desplazamiento se habrá de respetar la remuneración prevista en los países de destino, por más que la Unión no cuente con competencias para fijar salarios.

Pues bien, aun en el ámbito limitado de los supuestos de movilidad transnacional y añadiendo, como hace la propuesta de la Comisión, los supuestos de disrupción de ámbito europeo, la creación de la ELA puede ser un primer paso en este objetivo de asegurar las premisas o postulados básicos de la legislación social para este y otros contextos: es necesario determinar cómo asegurar la negociación colectiva —o la mediación, la huelga o cualquier otro derecho colectivo-, en su proceso y resultados, en transiciones de trabajo espaciales interestatales. No se trata solo, en este sentido, de dejar abierto el ejercicio de tales derechos según las prácticas nacionales, sino también de coordinar la aplicación de prácticas nacionales negociales distintas. Aunque desde un punto de vista material hubiera sido conveniente abordar expresamente esta cuestión respecto de la negociación colectiva y los pactos de huelga en la modificación de 2018 de la Directiva 96/71, también es posible abordar esta modificación posteriormente, conectándola, en su caso, con los Reglamentos sobre conflicto de referencia.

También es oportuno anticipar la necesidad de una mayor visibilidad y acción de la Unión en el ámbito de la igualdad y la no discriminación en relación con las distintas causas objeto de regulación — sexo, raza, libertad y orientación sexual, libertad religiosa, edad, entre otras- y de otras

${ }^{20}$ En relación con las propuestas de convenio colectivo a nivel europeo me remito a la Resolución del Parlamento Europeo 2012/2292 (INI), de 12 de septiembre de 2013, sobre las negociaciones colectivas transfronterizas y el diálogo social transnacional, y S. SCIARRA, M. FuchS y A. SobCZAK, «Hacia la adopción de un marco jurídico para los convenios colectivos transnacionales. Informe destinado a la Confederación Europea de Sindicatos», informe que está disponible en https://www.etuc.org/sites/www.etuc.org/files/publication/ files/140225_experts_report_tca_es_not_checked.pdf. 
causas conexas y contempladas en la Carta Europea de Derechos Fundamentales, ámbito donde la propuesta de constitución de la ELA no contiene, según puedo anticipar, referencias expresas por el momento, por más que los desarrollos internos a la ELA o paralelos en las reformas de directivas y reglamentos de libre circulación puedan asumir en un futuro esta función, siguiendo, en su caso, prácticas nacionales. Una referencia expresa, cuanto menos, a la labor de difusión de buenas prácticas y experiencias legales nacionales y de la negociación colectiva respecto de la defensa del papel de la mujer, del colectivo LGTBI, de menores y mayores, de extranjeros o de personas con capacidades especiales y necesidad de apoyo sería del todo oportuna.

$\mathrm{Al}$ margen de los anteriores ámbitos, existen otros donde la actividad transnacional de la Unión resulta más perceptible y se encuentra totalmente afianzada, como acontece con la Red Europea de Servicios de Empleo (EURES), regulada por el Reglamento (UE) 2016/589 del Parlamento y del Consejo, de 13 de abril de 2016, o la Red de Resolución de Problemas en el Mercado Interior (SOLVIT), ámbitos donde el propio objeto de la regulación hace imposible o muy difícil renunciar a una gestión coordinada - y plenamente visible en el nivel administrativo- por parte de organismos de la Unión Europea. Con la forma de Asociación entre Estados, la Comisión e Interlocutores Sociales, la Red EURES ha supuesto la creación de órganos europeos adscritos a la Comisión, como es la Oficina Europea de Coordinación, encargados de garantizar y desarrollar la intermediación laboral a nivel Europeo. La regulación vigente de esta red es todavía reciente, de 2016, y su objetivo ha sido reflejar las nuevas pautas de movilidad, la mayor exigencia de movilidad equitativa, los cambios en la tecnología de intercambio de datos sobre ofertas de empleo, el uso de distintos canales de información entre trabajadores y empresarios, y la importancia de otros agentes en el mercado de trabajo, junto con los servicios públicos ${ }^{21}$. Como veremos seguidamente, estos y otros órganos y objetivos vienen a ser integrados y coordinados por la nueva propuesta normativa.

${ }^{21}$ En relación con el alcance de la nueva regulación de EURES junto con las modificaciones operadas en materia de simplificación documental para facilitar la circulación de personas, me remito más recientemente a J. GarCía MurCia, «Dos nuevos reglamentos de la Unión Europea para facilitar la libre circulación (de trabajadores y de personas)», Foro. Nueva época, núm. 1 (2016), pp. 413-422. En relación con las coordenadas generales de la materia me remito a F. Valdés Dal-Ré y J. M. Zufiaur Narvaiza, Hacia un mercado europeo de empleo, Madrid, Ministerio de Trabajo e Inmigración, 2006. 
En relación singularmente con la Inspección de Trabajo y Seguridad Social, la normativa actual parte, en el ámbito específicamente laboral, de un reparto de competencias donde el país de destino se reserva la facultad de visitar los centros de trabajo de su territorio controlando el cumplimiento de la legislación, mientras que el país de origen sigue desarrollando también facultades de control de legalidad conforme a los distintos apartados del art. 7 de la Directiva 2014/67/CE 22 . Esta misma norma prevé el intercambio de información entre Administraciones, así como la posibilidad de una cooperación funcionarial a través de acciones de intercambio, formación, bases de datos o sitios web conjuntos u otras iniciativas similares. En la práctica, a través de convenios bilaterales entre Estados se han creado equipos conjuntos de inspección ${ }^{23}$; convenios a los que apela el art. 16.10 de la Ley 23/2015, de 21 de julio, Ordenadora del Sistema de Inspección de Trabajo y Seguridad Social ${ }^{24}$. Igualmente cabe citar también la actividad que viene desempeñando el Comité de Altos Responsables de la Inspección de Trabajo (CARIT) ${ }^{25}$ de fomento de cooperación entre Ins-

${ }^{22}$ Cfr. M. VÁzquez, «La vigilancia y control de la Inspección de Trabajo...», op. cit., pp. 262 y 263.

${ }^{23} \mathrm{Ibid}$., pp. 262 y 263 . Entre las principales deficiencias internas que se constatan por el autor está la ausencia de bases de datos creadas en sede administrativa que puedan ser consultadas por la Inspección de Trabajo. Así, en relación con el certificado A1, vid. M. L. VegA RuIz, Garantizando la gobernanza. Los sistemas de Inspección de Trabajo en el mundo. Tendencias y retos. Un enfoque comparado, Ginebra, OIT, 2013, pp. 166 y ss., y J. García MuRCIA (dir.), La inspección de trabajo: regulación española y perspectiva internacional, Aranzadi, Cizur Menor, 2016, pp. 522 y ss.

${ }^{24}$ En dicho apartado se establece que: «La colaboración de las autoridades de los Estados miembros de la Unión Europea con competencias equivalentes a las de la Inspección de Trabajo y Seguridad Social se regirá por la normativa de la Unión Europea o por los instrumentos o acuerdos bilaterales o multilaterales de los que sea parte el Estado español. Los hechos comprobados por dichas autoridades en el ámbito de la cooperación administrativa internacional que sean facilitados a las autoridades españolas podrán ser aducidos como prueba por la Inspección de Trabajo y Seguridad Social en los procedimientos iniciados por esta y serán tenidos por ciertos, salvo prueba en contrario de los interesados».

${ }_{25}$ Conforme a la Decisión de la Comisión de 12 de julio de 1995 (DOCE de 9 de agosto de 1995), por la que se crea un Comité de Altos Responsables de la Inspección de Trabajo. Este Comité asume: 1) definición de principios comunes de inspección de trabajo en materia de salud y seguridad en el lugar de trabajo y elaboración de métodos de evaluación de los sistemas nacionales de inspección en relación con dichos principios; 2) promoción de un mejor conocimiento y comprensión mutua de los diferentes sistemas y prácticas nacionales de inspección de trabajo, de los métodos y de los marcos jurídicos de intervención; 3) desarrollo de intercambios de experiencias entre los servicios nacionales de inspección del trabajo respecto al control de la aplicación del Derecho comunitario derivado en materia de salud y seguridad en el trabajo, a fin de asegurar su aplicación coherente en la Comunidad; 4) promoción de los intercambios de inspectores de trabajo entre las Administraciones nacionales y elaboración de programas de formación dirigidos a los inspectores; 
pecciones y desarrollo de buenas prácticas; actividad que, como veremos después, va a ser encabezada por la propia ELA. Estos desarrollos se realizan sobre una base reguladora internacional que aborda y trata de establecer estándares comunes sobre la inspección de trabajo tanto en el ámbito de la OIT, a través singularmente del Convenio 81 de 1947, como del Consejo de Europa, a través de la Carta Social Europea ${ }^{26}$.

En un terreno más fáctico es oportuno recordar que la creación de la ELA operaría sobre un escenario de más de diecisiete millones de personas que trabajan o viven en un Estado distinto al de su nacionalidad, como recuerda la propuesta de la Comisión Europea. De otro lado, por más que conocido, también es importante insistir en que la propuesta actúa en el contexto de la potenciación del Derecho social de la Unión a través del mencionado pilar social y un conjunto de iniciativas como las propuestas de modificación de las directivas sobre desplazamiento de trabajadores y los reglamentos de coordinación de la Seguridad Social, la regulación singular de los trabajadores del sector de transporte, las directivas para garantizar el cumplimiento de las reglas sobre movilidad, la nueva regulación de EURES o el establecimiento de la plataforma europea para abordar el trabajo no declarado.

Tras esta presentación es posible exponer el diagnóstico y los objetivos sobre los que descansa la propuesta. Cabe anticipar que nos encontramos ante un primer paso, muy relevante, en línea con las preocupaciones arriba expresadas. Las funciones de la autoridad en muchos ámbitos no son preceptivas —así, en materia de mediación en Seguridad Social— o se someten al acuerdo interestatal - por ejemplo, las inspecciones de trabajo conjuntas — o a la solicitud de la Administración — por ejemplo, en materia de mediación en casos de reestructuración-.

5) elaboración y publicación de documentos destinados a facilitar la actividad de los inspectores de trabajo; 6) desarrollo de un sistema fiable y eficaz de intercambio rápido de información entre las inspecciones de trabajo sobre cualquier problema que pudiera plantear el control de la ejecución de la legislación de origen comunitario en el ámbito de la salud y la seguridad en el lugar de trabajo; 7) establecimiento de una cooperación activa con las inspecciones de trabajo de terceros países a fin de promover el trabajo realizado por la Comunidad en materia de salud y seguridad en el trabajo y facilitar la resolución de posibles problemas transfronterizos; 8) estudio del posible efecto de otras políticas comunitarias en las actividades de las inspecciones de trabajo relativas a la salud y seguridad en el trabajo y a las condiciones de trabajo.

${ }^{26}$ Sobre esta labor me remito a F. VÁzQuez, La Carta Social Europea y la Inspección de Trabajo. Un estudio comparado de los ordenamientos español, portugués y francés por la vía del procedimiento de control de informes, Barcelona, Atelier, 2018. 


\section{DIAGNÓSTICO Y OBJETIVOS}

Las anteriores pautas introductorias tratan de dar cuenta de las necesidades específicas que plantea la aplicación del Derecho social europeo en supuestos de movilidad transnacional. Son ya, de por sí, un diagnóstico que surge desde un análisis de las características propias de la rama social del ordenamiento. Junto con estas necesidades existen otras, más generales, que tienen que ver con la garantía de aplicación y la efectividad de las normas, que son consideradas ampliamente en la propuesta de la Comisión, así como con los presupuestos, condiciones o capacidades que hacen posible el ejercicio de los derechos o, si se prefiere, que pueden asociarse a la idea de Derecho.

En efecto, entre las principales preocupaciones expuestas por la Comisión, como veremos seguidamente de forma sistemática, se encuentra la garantía de fácil acceso a las regulaciones nacionales y de la Unión aplicables para evitar que la incertidumbre sea un límite a la movilidad y a la búsqueda de empleo. Igualmente se quiere evitar, una vez asumida la movilidad, que esta suponga una merma de derechos o ventajas, en forma de trabajo minusvalorado, para empresas y trabajadoras o trabajadores; en fin, se quiere evitar también la inseguridad asociada a un marco jurídico disperso o de difícil acceso. Nos situamos, sin embargo, ante una propuesta que se mueve básicamente en un terreno orgánico y procedimental, y que debe estar vinculada ineludiblemente con el replanteamiento de diversos ámbitos sustantivos, como viene haciéndose en las distintas propuestas de modificación de directivas y reglamentos, singularmente la Directiva 96/71/CE, modificada recientemente, como se ha advertido, o el Reglamento 883/2004.

En este contexto, el diagnóstico explícito realizado por la Comisión para justificar la creación de la ELA se desdobla en las siguientes consideraciones generales: a) la necesidad de mejorar el asesoramiento y apoyo a trabajadoras, trabajadores y empresas sobre el trabajo transnacional, especialmente en materia de información sobre derechos y obligaciones; b) la necesidad de mejorar el acceso a información compartida entre autoridades nacionales en los diferentes ámbitos de la movilidad y la coordinación de la Seguridad Social; c) la necesidad de mejorar la capacidad de las autoridades nacionales para organizar la cooperación; d) la necesidad de mejorar los mecanismos para llevar a cabo actividades de aseguramiento e inspección de la aplicación de la legislación; e) la necesidad de crear meca- 
nismos de mediación entre Estados miembros en relación con los distintos dominios de la movilidad laboral y la coordinación de la Seguridad Social; f) la mejora de cooperación a nivel UE que garantice una justa movilidad en el mercado interior. Cada una de estas constataciones da pie a un capítulo o ámbito de propuestas, según veremos a continuación. Se trata, en cualquier caso, de avanzar sobre las dificultades constatadas y, sobre todo, hacerlo a través de un tratamiento conjunto o coordinado en el ámbito de un único organismo, la ELA.

Debe también constatarse de inmediato que la propuesta, como la propia proclamación del pilar social europeo, asume la misión fundamental de garantizar los derechos preexistentes más que, en principio, crear nuevos derechos. En este sentido, tal y como se indica, la regulación propuesta debe contribuir a la implementación de dicho pilar mediante una efectiva y eficiente garantía de aplicación del Derecho de la Unión en materia de movilidad y Seguridad Social, así como en relación con los convenios y acuerdos colectivos que implementan tal Derecho de la Unión. De otro lado, se trata de promover el conocimiento de las oportunidades de formación y aprendizaje a largo plazo, así como un apoyo activo al empleo. Sin perjuicio de ello, no cabe duda de que la certeza en la forma en que pueden ejercerse cualesquiera derechos laborales es una garantía ineludible para su ejercicio, cuando no una forma explícita de ser de esos mismos derechos, especialmente si entre esas formas o modalidades de ejercicio se incluyen los elementos temporal y espacial o se abordan cuestiones como la coordinación y sucesión de varios ordenamientos jurídicos distintos.

\section{PRINCIPALES ÁMBITOS DE ACTUACIÓN EUROPEOS: EMPLEO, SEGURIDAD SOCIAL, INSPECCIÓN DE TRABAJO, MEDIACIÓN ENTRE ADMINISTRACIONES, MEDIACIÓN EN CASOS DE REESTRUCTURACIÓN}

$\mathrm{El}$ articulado de la propuesta aborda en primera instancia las funciones de la ELA, definiendo de forma sucesiva sus distintos objetivos y ámbitos de actuación. Sus actividades, conforme al art. 5, tratan de ser coherentes - y son prácticamente simétricas - con los objetivos propuestos, enunciados en el anterior apartado. En cualquier caso, como el art. 1 indica, nos encontramos ante competencias vinculadas con la movilidad transnacional y la coordinación de los sistemas de Seguridad Social. Estas competencias son las siguientes: 
a) Facilitar el acceso a particulares y empresas al ejercicio de los derechos y obligaciones concurrentes en casos de movilidad transfronteriza.

b) Facilitar la cooperación y el intercambio de información entre autoridades nacionales para asegurar la aplicación de Derecho de la Unión.

c) Coordinar y prestar apoyo a las inspecciones concertadas y conjuntas.

d) Llevar a cabo análisis y valoraciones de riesgo sobre asuntos vinculados a la movilidad transnacional.

e) Reforzar las capacidades de los Estados para asegurar el efectivo cumplimiento y ejecución del Derecho de la Unión pertinente.

f) Mediar entre Estados miembros en relación con la aplicación del Derecho pertinente de la Unión.

g) Facilitar la cooperación entre grupos de interés y agentes sociales en casos de transformación o reestructuración transnacionales.

Siguiendo este orden expositivo, los elementos o cuestiones en materia de empleo más destacables parten de la designación de la ELA como gestor y director de la Oficina de Coordinación Europea de EURES, pasando esta Oficina a integrarse en la ELA, sin perjuicio de que la dependencia de los servicios informáticos siga asociada a la Comisión Europea. Respecto del alcance de la funciones, cabe destacar que estas incluyen las propias de la intermediación laboral, así como las de estudio, en colaboración con otros órganos y redes, de los obstáculos sobre la movilidad y también la información y difusión de información entre particulares y Estados —con apoyo a unos y a otros- sobre la legislación social aplicable, estatal y de la Unión, y sobre los recursos materiales necesarios para acceder a la actividad laboral (cursos de idiomas, servicios de salud o, entre otras, condiciones de vida y trabajo).

Respeto del intercambio de información entre autoridades, la ELA pasa a convertirse en un intermediario y facilitador de la comunicación, de forma tal que no sustituye los procedimientos y obligaciones actualmente concurrentes, sino que trata de crear un canal de apoyo para el seguimiento de las solicitudes de información realizadas, para el desarrollo de actividades vinculadas (como la traducción de textos) o para la facilitación del cumplimiento de procedimientos sancionadores. No se modifican, por tanto, las disposiciones concurrentes en las Directivas 2014/67/CE y 2014/54/CE en relación con el intercambio de información entre autori- 
dades y la actuación de la Inspección de Trabajo. Respecto de los medios técnicos actualmente vigentes, y según se indica literalmente en los considerandos iniciales de la propuesta, la ELA fomentará el uso de aproximaciones novedosas a una efectiva y eficiente cooperación, incluyendo las herramientas de intercambio de información como el mecanismo de Intercambio Electrónico de Información de Seguridad Social (ESSI por sus siglas en inglés) y el Sistema de Información del Mercado Interior (IMI por sus siglas en inglés), y promoverá la mejora de las herramientas y procedimientos tecnológicos para intercambiar información entre autoridades nacionales (considerando 13).

Las anteriores actividades de fomento de la cooperación y el desarrollo de nuevas medidas y procedimientos pueden conectarse también, de un modo general, con las expectativas de colaboración que se contemplan en la propuesta con otros órganos y redes de la Unión preexistentes, como, entre otras, la Red Europea de Servicios Públicos de Empleo, el ya mencionado SOLVIT, la Agencia Europea de Seguridad y Salud en el Trabajo (OSHA), el Centro Europeo de Formación Profesional (CEDEFOP) y la Fundación Europea para la Mejora de las Condiciones de Vida y de Trabajo (EUROFOUND).

Destaca también, en tercer lugar, la coordinación de inspecciones de trabajo conjuntas, que puede ser desarrollada a instancias de cualquier de las autoridades nacionales o de la propia ELA y que requieren, en cualquier caso, el acuerdo de los distintos Estados implicados —al menos de dos si son tres o más-. Estas opciones, como se ha visto, han venido descansando en el desarrollo de acuerdos bilaterales.

Igualmente, en orden a prestar apoyo a la actividad de los Estados, se prevé el desarrollo de guías comunes de actuación en el ámbito de la inspección y administración de trabajo, de difusión de buenas prácticas —especialmente en materia de cooperación entre autoridades- y el desarrollo de programas sectoriales de formación, de forma tal que la ELA pasa a asumir un papel directivo en algunas de las principales actividades anteriormente desempeñadas por el CARIT. Las limitaciones en esta función de cooperación en la actuación de la ELA no han impedido que se le atribuya la posibilidad de comunicar a la Comisión Europea y a las autoridades nacionales cualquier incumplimiento.

La difusión de buenas prácticas también alcanza al denominado trabajo no declarado, pasando la autoridad a asumir las funciones de desarrollo y apoyo de buenas prácticas, formación e intercambio de información que son actualmente atribuidas a la plataforma del mismo nombre. 
Por su parte, en materia de valoración de los problemas vinculados a la movilidad transnacional, la ELA desarrollará una labor de estudio de los desequilibrios o amenazas sectoriales que pueden surgir, así como sobre los problemas recurrentes de la movilidad, actuando de forma complementaria con otras agencias y servicios de la Unión. Llevará a cabo también una labor de compilación de los datos facilitados por los Estados miembros en materia de Derecho de la Unión.

En siguiente lugar, la ELA asumirá una función de mediación interestatal tanto en materia de Seguridad Social — pasando a desarrollar parcialmente las funciones de la Comisión Administrativa prevista en los Reglamentos 883/2004 y 987/2009- como laboral a iniciativa de los Estados o planteada a través de SOLVIT, si los Estados así lo acuerdan. También puede plantearse a instancias de la ELA. En relación específicamente con la coordinación de la Seguridad Social, la ELA asume, como se dice, las funciones de mediación que actualmente desempeña la Comisión Administrativa, a la que en el resto de actividades pasa a prestar apoyo y complementar en relación con las discrepancias que puedan surgir entre autoridades sobre la aplicación de su legislación de Seguridad Social en casos de desplazamientos y movilidad laboral. No se establece el carácter vinculante de las soluciones alcanzadas, por otra parte extraño a la idea de mediación.

La ELA también tiene encomendada una función de soporte y ayuda para el desarrollo de la capacidad de los Estados miembros para garantizar el cumplimiento de la normativa aplicable, singularmente respecto de la asistencia entre autoridades, el desarrollo de guías de uso administrativo o el desarrollo de programas sectoriales de formación.

Por último, a solicitud de las autoridades nacionales, la ELA podrá desarrollar una labor de facilitación de la cooperación entre los agentes sociales y grupos de interés en relación con transformaciones del mercado de trabajo que afectan a más de un Estado miembro, como reestructuraciones de amplio alcance o eventos relevantes que afectan a regiones fronterizas. Esta competencia, tan atractiva o sugerente como las demás, es definida de forma muy abierta y escueta, pero su lectura nos permite vislumbrar la creación de un órgano de mediación o conciliación a nivel europeo partiendo de una función inicial de reconocimiento de interlocutores y facilitación del diálogo. No se aclara, sin embargo, como se apuntó arriba, si esta posibilidad debe vincularse exclusivamente con la movilidad transfronteriza, partiendo del alcance aplicativo general de la propuesta que se define inicialmente. 


\section{ORGANIZACIÓN DE LA ELA Y ÓRGANOS COMPLEMENTARIOS}

La organización de la ELA incluye expresamente órganos de ámbito europeo, si bien estos deben conectarse con órganos preexistentes que integra o con los que pasa a colaborar, así como con los agentes nacionales. Salvo los primeros, los seguidamente citados no son directamente regulados por la propuesta, sino que están integrados en la regulación de los órganos que la ELA pasa a sustituir total o parcialmente en el ámbito europeo, o a dirigir y coordinar, como acontece con las oficinas nacionales de coordinación de EURES. Entre los órganos europeos específicos o propios de la ELA nos encontramos con el Comité de Dirección, integrado por un representante senior y un sustituto de cada Estado miembro y dos miembros de la Comisión, todos con derecho de voto y elegidos cada cuatro años susceptibles de prórroga; un director ejecutivo, designado por el Comité de Dirección, que responde ante el Parlamento Europeo y actúa como representante legal de la ELA, y un Grupo de Agentes Sociales, integrado por seis representantes de los agentes sociales a nivel de la Unión y dos representantes de la Comisión, cuyas funciones básicas son de tipo consultivo. Se prevé, por otra parte, la creación de grupos de trabajo y paneles específicos para desarrollar tareas concretas. La propuesta normativa contempla finalmente las habituales consignaciones presupuestarias y relativas a la plantilla de la ELA y remite el desarrollo de buena parte de estos aspectos a una futura regulación por parte de sus propios órganos.

Por último, la propuesta prevé la desaparición de diversos órganos y comités actualmente existentes, como el Comité Técnico relativo a la Libre Circulación de Trabajadores ${ }^{27}$, el Comité de Expertos sobre Desplazamiento de Trabajadores ${ }^{28}$, el Comité Consultivo, el Comité de Auditoría y el Comité de Conciliación en el ámbito de la Coordinación de los Sistemas de Seguridad Social ${ }^{29}$ y la Plataforma Europea para abordar el Trabajo no Declarado ${ }^{30}$.

${ }^{27}$ Reglamento UE 492/2011 del Parlamento y del Consejo, de 5 de abril de 2011, relativo a la libre circulación de los trabajadores dentro de la Unión (DOUE de 27 de mayo de 2011).

${ }^{28}$ Decisión de la Comisión 2009/17/CE, de 19 de diciembre de 2008, por la que se crea el Comité de Expertos sobre desplazamiento de trabajadores (DOUE de 13 de enero de 2009).

${ }_{29}$ Decisión núm A1, de 12 de junio de 2009, relativa al establecimiento de un procedimiento de diálogo y conciliación sobre la validez de los documentos, la determinación de la legislación aplicable y el abono de prestaciones de acuerdo con el Reglamento (CE) núm. 883/2004 del Parlamento Europeo y del Consejo (DOUE de 24 de abril de 2010).

30 Decisión UE 2016/344 del Parlamento y del Consejo, de 9 de marzo de 2016, por la que se establece la plataforma europea para mejorar la cooperación en el tratamiento del trabajo no declarado (DOUE de 11 de marzo de 2016). 